\title{
BIBLIOGRAPHY
}

1. Claude Chevalley, Theory of Lie groups, Princeton University Press, 1946.

2. C. N. Lee, Covering spaces and simple connectedness, Duke Math. J. vol. 24 (1957) pp. 547-554.

3. R. S. Novosad, Simply connected spaces, Trans. Amer. Math. Soc. vol. 79 (1955) pp. 216-228.

University OF Minnesota AND

UNIVERSITY OF WASHINGTON

\section{ONE DIMENSIONAL TOPOLOGICAL LATTICES}

LEE W. ANDERSON ${ }^{1}$

1. By a topological lattice we mean a Hausdorff space $L$ and a pair of continuous functions $\bigvee: L \times L \rightarrow L$ and $\wedge: L \times L \rightarrow L$ which satisfy the usual conditions stipulated for a lattice in Birkhoff [2, p. 18]. The purpose of this paper is to prove the

Main Theorem. A locally compact, connected, one dimensional topological lattice is a chain.

The lattice theoretic terminology used in this paper is consistent with Birkhoff [2]. The topological terms can be found in [5] or [8] with the following exceptions. If $X$ and $Y$ are sets, $X \backslash Y$ denotes the relative compliment of $Y$ with respect to $X$. If $A$ is a subset of a topological space then $A^{*}, A^{0}$ and $F(A)=A^{*} \backslash A^{0}$ denote the topological closure, interior and boundary of $A$. The symbol $\varnothing$ denotes the empty set.

We will agree that in usage of words common to topology and lattice theory, the topological meaning will take precedence. Thus to say that a subset $A$ of a topological lattice is closed means $A=A^{*}$ and not $A \wedge A \subset A$ or $A \vee A \subset A$.

If $L$ is a lattice and $A$ is a subset of $L$ we let

$$
C(A)=(A \wedge L) \cap(A \vee L) \text {. }
$$

If $A=C(A)$ we say that $A$ is a convex subset of $L$. It is clear that the set $A$ is convex if, and only if, $x \vee(y \wedge L) \subset A$ whenever $x$ and $y$ are elements of $A$ with $x \leqq y$. A topological lattice is locally convex if, and only if, whenever $x$ is an element of an open set $U$ there is an open convex set $V$ with $x \in V \subset U$.

Received by the editors October 1, 1955 and, in revised form, April 21, 1958.

1 This work was supported in part by a National Science Foundation Grant. 
We reserve the symbols 0 and 1 to denote the minimal and maximal elements of a lattice whenever they exist.

Examples of topological lattices may be found in [1] or [4]. The Euclidean plane with the usual topology and coordinatewise lattice operations is a simple but useful example of a topological lattice.

The author is indebted to Professor A. D. Wallace for his advice during the preparation of this paper.

2. L. Nachbin [6] showed that a compact partially ordered topological space is locally convex. In this section we modify his result and prove

Theorem 1. A locally compact, connected, topological lattice is locally convex.

Some preliminary lemmas are in order before we proceed with the proof of Theorem 1.

LEMma 1. If $L$ is a connected topological lattice and if $A$ is a subset of $L$ with $A \wedge L \subset A$ or $A \vee L \subset A$ then $A$ is connected.

Proof. If $a \in L$ then the function $f(x)=a \wedge x$ is continuous and so $f(L)=a \wedge L$ is a connected set. Now if $A \wedge L \subset A$ and if $x$ and $y$ are elements of $A$ then $x \wedge L \subset A$ and $y \wedge L \subset A$. Also $x \wedge L$ and $y \wedge L$ are connected sets with $(x \wedge L) \cap(y \wedge L) \neq \varnothing$ and so $(x \wedge L) \cup(y \wedge L)$ is a connected subset of $A$ which contains both $x$ and $y$. Therefore $A$ is connected.

Lemma 2. If $L$ is a topological lattice and if $U$ is an open subset of $L$ then $U \wedge L$ and $U \vee L$ are open.

Proof. We will show that $U \wedge L$ is open. Suppose $\left\{x_{\alpha}\right\}$ is a net in $L \backslash(U \wedge L)$ and $\left\{x_{\alpha}\right\}$ converges to some element $y$ in $L$. If $y \in U \wedge L$ then for some $z \in U, y \wedge z=y$. Now $\vee$ is a continuous function and so the net $\left\{x_{\alpha} \vee z\right\}$ converges to $y \vee z=z$ and $z$ is an element of $U$. Since $U$ is open, there is an $x_{\alpha}$ such that $x_{\alpha} \vee z \in U$ and so $x_{\alpha}=\left(x_{\alpha} \vee z\right)$ $\wedge x_{\alpha} \in U \wedge L$ which is a contradiction. Thus $y \in L \backslash(U \wedge L)$ and therefore $L \backslash(U \wedge L)$ is closed which implies that $U \vee L$ is open. A dual argument will show that $U \bigvee L$ is open

Lemma 3. If $L$ is a topological lattice and if $A \subset L$ is compact then $A \wedge L$ and $A \vee L$ are closed.

Proof. We will show that $A \wedge L$ is closed. Suppose $x$ is not an element of $A \wedge L$. Then $x \wedge A$ is a compact set which does not contain $x$, hence there are disjoint open sets $U$ and $V$ such that $x \in U$ and $x \wedge A \subset V$. Now $\wedge$ is continuous and $A$ is compact, hence there is an 
open set $W$ such that $x \in W$ and $W \wedge A \subset V$. Now if $y \in(W \cap U)$ $\cap(A \wedge L)$ then for some $a \in A, y \wedge a=y$ and therefore $y=a \wedge y \in A$ $\wedge W \subset V$ and $y \in W \cap U \subset U$ which contradicts the choice of $U$ and $V$. Thus $W \cap U$ is an open subset of $L \backslash(A \wedge L)$ which contains $x$ and so it follows that $A \wedge L$ is closed. Dually we get $A \vee L$ closed.

Combining Lemmas 2 and 3 we have

Lemma 4. If $L$ is a topological lattice and if $A$ is a subset of $L$, then

(i) $C(A)$ is closed whenever $A$ is compact.

(ii) $C(A)$ is open whenever $A$ is open.

We now prove Theorem 1. Let us assume that $a$ is an element of an open subset $U$ of $L$. We must show there is an open set $V$ with $a \in C(V) \subset U$. To this end, let us assume that this proposition is false, i.e. that $(L \backslash U) \cap C(V) \neq \varnothing$ for all open sets $V$ which contain $a$. Now $L$ is locally compact, hence there is an open set $P$ such that $a \in P$ $\subset P^{*} \subset U$ and $P^{*}$ is compact. Let $W$ denote the collection of all open sets $W \subset L$ such that $a \in W \subset W^{*} \subset P$. Since we have assumed that $C(W) \cap(L \backslash U) \neq \varnothing$ for all $W \in W$, it follows that $C\left(W^{*}\right) \cap(L \backslash P) \neq \varnothing$ for all $W \in W$. We will now show that $C\left(W^{*}\right) \cap F(P) \neq \varnothing$ for all $W \in W$. If $W \in W$ and if $z \in C\left(W^{*}\right) \cap(L \backslash P)$ then for some $x \in W^{*}$ we have $z=z \wedge x$ and therefore $x, z \in z \bigvee(x \wedge L) \subset C\left(W^{*}\right)$. By Lemma 1 , $z \bigvee(x \wedge L)$ is connected and since $z \bigvee(x \wedge L)$ meets both $P$ and $L \backslash P, \quad(z \vee(x \wedge L)) \cap F(P) \neq \varnothing$. But $z \bigvee(x \wedge L) \subset C\left(W^{*}\right)$ and so $C\left(W^{*}\right) \cap F(P) \neq \varnothing$. From the regularity of $L$ it follows that the collection of closed sets $\left\{C\left(W^{*}\right) \cap F(P): W \in W\right\}$ has the finite intersection property. Therefore, since $P^{*}$ is compact, we have $\cap\left\{C\left(W^{*}\right) \cap F(P): W \in W\right\} \neq \varnothing$. However, it is easily seen that $\cap\left\{C\left(W^{*}\right): W \in W\right\}=(a \wedge L) \cap(a \vee L)=\{a\}$ and so $\{a\}$ $=\bigcap\left\{C\left(W^{*}\right) \cap F(P): W \in W\right\} \subset F(P)$ which is a contradiction. Hence the result is established.

3. In this section we prove the main theorem. We first prove a few preliminary results.

Lemma 5. If $L$ is a topological lattice and if $a \in L$ then $(a \wedge L)$ $\bigvee F(a \wedge L)=F(a \wedge L)$ and $(a \bigvee L) \wedge F(a \bigvee L)=F(a \bigvee L)$

Proof. Since $(a \wedge L) \bigvee[L \backslash(a \wedge L)] \subset L \backslash(a \wedge L)$ and $\vee$ is continuous, it follows that $(a \wedge L) \vee[L \backslash(a \wedge L)]^{*} \subset[L \backslash(a \wedge L)]^{*}$. Also $(a \wedge L) \vee(a \wedge L) \subset(a \wedge L)$ and therefore $(a \wedge L) \vee F(a \wedge L)$ $=(a \wedge L) \bigvee\left[(a \wedge L) \cap(L \backslash(a \wedge L))^{*}\right] \subset(a \wedge L) \cap[L \backslash(a \wedge L)]^{*}=F(a \wedge L)$. Since $\vee$ is an idempotent function and $F(a \wedge L) \subset a \wedge L$ we have $F(a \wedge L) \subset(a \wedge L) \vee F(a \wedge L)$ and so the result is established. A dual argument establishes that $(a \bigvee L) \wedge F(a \bigvee L)=F(a \bigvee L)$. 
If $L$ is a lattice and if $a \in L$, let

$$
U(a)=L \backslash[(a \wedge L) \cup(a \vee L)] .
$$

Clearly $U(a)$ is the set of elements in $L$ that are not comparable to $a$.

Lemma 6. If $L$ is a connected topological lattice and if $a \in L$ then

(i) $F(a \wedge L)$ and $F(a \bigvee L)$ are connected.

(ii) $a \wedge[L \backslash(a \wedge L)] \subset F(a \wedge L)$ and $a \vee[L \backslash(a \bigvee L)] \subset F(a \vee L)$

(iii) $a \in U(a)^{*}$ whenever $U(a)$ is not void.

Proof of (i). Clearly $a \wedge L$ is a sublattice of $L$ and by Lemma 1 , $a \wedge L$ is connected. Now by Lemma $5, F(a \wedge L) \bigvee(a \wedge L)=F(a \wedge L)$ and so by Lemma 1 , it follows that $F(a \wedge L)$ is connected. A dual argument shows that $F(a \bigvee L)$ is connected.

Proof of (ii). If $x$ is an element of $L \backslash(a \wedge L)$ then $x \wedge L$ contains both $x$ and $a \wedge x$, hence meets both $a \wedge L$ and $L \backslash(a \wedge L)$. Since $x \wedge L$ is a connected set, it follows that $x \wedge L$ also meets $F(a \wedge L)$. Let $y \in(x \wedge L) \cap F(a \wedge L)$ then $x \wedge y=y$ and $a \wedge y=y$ and so $(a \wedge x) \wedge y=y$ which implies that $(a \wedge x) \bigvee y=a \wedge x$. Now by Lemma 5 , we have $(a \wedge x) \vee y \in F(a \wedge L)$ and so $a \wedge x \in F(a \wedge L)$. Dually, we establish that $a \bigvee[L \backslash(a \bigvee L)] \subset F(a \bigvee L)$.

Proof of (iii). If $b$ is an element of $U(a)$ then $a \wedge b$ is an element of $F(a \wedge L)$ and $a \wedge b \neq a$. Since $F(a \wedge L)$ is connected and contains points other than $a$, it follows that $a$ is an accumulation point of $F(a \wedge L)$. Now if $V$ is an open set containing $a$, then $V \cap(L \backslash(a \bigvee L))$ $\cap F(a \wedge L)=V \cap(F(a \wedge L) \backslash\{a\}) \neq \varnothing$. Therefore we have that $V \cap(L \backslash(a \wedge L)) \cap(L \backslash(a \bigvee L))=V \cap U(a) \neq \varnothing$ and so $a \in U(a)^{*}$.

If $X$ is a space and $A$ a subset of $X$, we denote by $H^{n}(X, A)$ the $n$-dimensional Alexander-Kolomogoroff cohomology group of $X$ modulo $A$ with coefficients in some nontrivial abelian group with identity $e$. We note that $H^{n}(X, \varnothing) \equiv H^{n}(X)$. We recall that a locally compact Hausdorff space $X$ has codimension at most $n$ if, and only if, for each compact $A \subset X$ and each closed $B \subset A$ if $i: B \rightarrow A$ is the injection function then the induced homomorphism $i^{*}: H^{n}(B) \rightarrow H^{n}(A)$ is onto. For the essential properties of this dimension function, the reader is referred to $[3]$.

Lemma 7. A compact connected topological lattice of codimension at most one is a chain.

Proof. Let $L$ be a compact connected topological lattice of codimension at most one and suppose $L$ contains two unrelated elements, $a$ and $b$. By the Hausdorff Maximality Principle, there are maximal chains $M$ and $N$ which contain $a$ and $b$ respectively. Since 
$L$ is compact and connected, both $M$ and $N$ are closed, connected and contain 0 and 1 [9]. It is also easily seen that any proper subset of $M($ or $N$ ) which contains 0 and 1 is not connected.

We now consider the following Mayer-Vietoris exact sequence [8] (we denote the set $\{1\}$ by 1 )

$$
\begin{aligned}
H^{0}(M, 1) \times H^{0}(N, 1) & \stackrel{J^{*}}{\rightarrow} H^{0}(M \cap N, 1) \stackrel{\Delta^{*}}{\longrightarrow} H^{1}(M \cup N, 1) \\
& \stackrel{I^{*}}{\rightarrow} H^{1}(M, 1) \times H^{1}(N, 1) .
\end{aligned}
$$

It is proven in [7] that the groups of a compact connected topological lattice vanish in all dimensions greater than 0 , thus we have $H^{1}(M, 1)$ $=H^{1}(N, 1)=e$. Since $M$ and $N$ are connected, it follows that $H^{0}(M, 1)$ $=H^{0}(N, 1)=e$ and therefore $\Delta^{*}$ is an isomorphism onto. Now $M \cap N$ is a proper subset of $M$ which contains 0 and 1 , therefore $M \cap N$ is not connected. Thus it follows that $H^{0}(M \cap N, 1) \neq e$ and so $H^{1}(M \cup N) \approx H^{1}(M \cup N, 1) \neq e$. Also, $L$ is a compact connected topological lattice and so by $[7], H^{1}(L)=e$. However, the codimension of $L$ is at most one and $M \cup N$ is a closed subset of $L$, therefore if $i: M \cup N \rightarrow L$ is the injection function, then $i^{*}: H^{1}(L) \rightarrow H^{1}(M \cup N)$ is onto which is a contradiction.

We note that Lemma 7 apparently requires the use of algebraic topology. At least the author knows no purely set theoretic proof of this lemma.

MaIn Theorem. A locally compact connected topological lattice of codimension at most one is a chain.

Proof. Suppose $L$ is a locally compact connected topological lattice with codimension at most one and that $a$ is an element of $L$ with $U(a)$ not empty. By Theorem $1, L$ is locally convex and so there is an open convex set $V$ containing $a$ with $V^{*}$ compact. Since $\vee$ and $\wedge$ are continuous and $a \bigvee a=a=a \wedge a$, there is an open set $W$ containing $a$ with $W \backslash W \subset V$ and $W \wedge W \subset V$. By Lemma 6 , we have $a \in U(a)^{*}$ and so $W \cap U(a) \neq \varnothing$. Let $b \in W \cap U(a)$ so that $a \wedge b \in W \wedge W \subset V$ and $a \bigvee b \in W \vee W \subset V$. Letting $B=(a \wedge b) \vee((a \vee b) \wedge L)$ we have $B C V$ since $V$ is convex. Also $B$ is a closed subset of $V^{*}$, hence compact. Clearly $B$ is a sublattice of $L$ and, by Lemma $1, B$ is connected, therefore $B$ is a compact connected sublattice of $L$. Now $B$ is closed and the codimension of $L$ is at most one, hence the codimension of $B$ is at most one. Therefore by Lemma $7, B$ is a chain but this is contradiction since $B$ contains both $a$ and $b$. 


\section{REFERENCES}

1. L. W. Anderson, On the distributivity and simple connectivity of plane topological lattices, Trans. Amer. Math. Soc. vol. 91 (1959) pp. 102-112.

2. G. Birkhoff, Lattice theory, rev. ed., Amer. Math. Soc. Colloquium Publications, New York, 1948.

3. H. Cohen, A cohomological definition of dimension for locally compact Hausdorff spaces, Duke Math. J. vol. 24 (1954) pp. 209-224.

4. D. E. Edmondson, A nonmodular compact connected topological lattice, Proc. Amer. Math. Soc. vol. 7 (1956) p. 1157.

5. J. L. Kelley, General topology, New York, Van Nostrand, 1955.

6. L. Nachbin, Topologia e ordem, Chicago, 1950.

7. A. D. Wallace, Cohomology, dimension, and mobs, Summa Brasil Math. vol. 3 (1953) pp. 43-54.

8. - Unpublished notes, Tulane University, 1952.

9. L. E. Ward, Jr., Partially ordered topological spaces, Proc. Amer. Math. Soc. vol. 5 (1954) pp. 144-161.

10. - Binary relations in topological spaces, An. Acad. Brasil Ci. vol. 26 (1954) pp. 357-373.

Tulane University AND

UNIVERSITY OF OREGON 